\title{
Culinary Properties of Raw Versus Conventional Soy Sauce during Tuna Preparation
}

\author{
Mami Ando ${ }^{1}$, Akio Obata ${ }^{2}$, Wen Jye Mok $^{3} \&$ Satoshi Kitao ${ }^{4}$ \\ ${ }^{1}$ Department of Food and Nutrition, Faculty of Agriculture, Setsunan University, Osaka, Japan \\ ${ }^{2}$ Research and Development Division, Kikkoman Corporation, Chiba, Japan \\ ${ }^{3}$ Institute of Marine Biotechnology, Universiti Malaysia Terengganu, Terengganu, Malaysia \\ ${ }^{4}$ Faculty of Health and Nutrition, Osaka Shoin Women's University, Osaka, Japan \\ Correspondence: Mami Ando, Department of Food and Nutrition, Faculty of Agriculture, Setsunan University, \\ 45-1 Nagaotoge, Hirakata, Osaka, 5730101, Japan. Tel: 81-72-896-6309. E-mail: mami.ando@ setsunan.ac.jp
}

Received: September 15, 2021

Accepted: December 10, 2021

Online Published: December 22, 2021

doi:10.5539/jfr.v11n1p10

URL: https://doi.org/10.5539/jfr.v11n1p10

\begin{abstract}
Soy sauce is a traditional Japanese seasoning made from fermented soybeans. As global demand grows, identifying novel soy sauce applications and benefits must become a priority. While conventional soy sauce undergoes heat-sterilization, filter-sterilization produces a lighter-colored (raw) soy sauce with preserved mold enzyme activities. As the impact of raw soy sauce during food (especially seafood) preparation remains unstudied, the present study compared the differential impact of raw and conventional soy sauce on tuna culinary properties. First, soy sauce color and protease activity were assessed. Next, tuna was marinated in soy sauce and non-alcoholic mirin for $0,10,35$, or 60 min. Finally, marinated tuna properties (mass, salt content, surface salt penetration, color, rupture load, surface wetness, and protein content) were objectively assessed, and subjective sensory evaluation (appearance, aroma, wetness, softness, saltiness, umami, and overall taste) was performed by a blinded panel. Findings confirmed the lighter color of and the preservation of protease activity in raw soy sauce. Raw soy sauce significantly enhanced surface tenderization, salt penetration, and wetness, while both soy sauces increased surface firmness via salt-induced dehydration. Respondents significantly preferred the appearance and saltiness level of raw soy sauce-marinated tuna, and the umami and overall taste of tuna marinated in raw soy sauce for $60 \mathrm{~min}$. The findings of this study, to our knowledge, demonstrate for the first time the potential culinary superiority of raw soy sauce in certain applications, and support future research to further define such applications.
\end{abstract}

Keywords: culinary science, filter-sterilization, heat-sterilization, meat tenderization, proteases, seasoning, soy sauce, tuna

\section{Introduction}

Soy sauce, made from fermented soybean paste, is a traditional Japanese seasoning. It has now become a standard ingredient around the world and in 2019, Japanese soy sauce exports reached a record high of 37100 kL (to 71 countries) (Soy Sauce Information Center, 2019; https://www.soysauce.or.jp/statistical-data). Many soy sauce varieties are available globally, including raw soy sauce.

Conventionally, soy sauce is heat-sterilized: during the final stage of production, soy sauce is heated to $70-90{ }^{\circ} \mathrm{C}$ for up to several dozen hours in order to kill the koji mold, yeast, and lactic acid bacteria used during the fermentation process; heating also standardizes quality (Nojiro, Yoshizawa, Kamada, Mizunuma \& Tatenuma, 1991; Higashi, 2008). Heating induces Maillard reaction, producing the distinctive aroma and dark reddish-purple color of soy sauce (Nojiro et al., 1991; Higashi, 2008). However, production of raw soy sauce employs filtration rather than heat sterilization, preventing an extensive occurrence of the Maillard reaction and resulting in a lighter color than that of the conventionally manufactured soy sauces (Martins, Jongen \& Van Boekel, 2000; Obata \& Kuwagaki, 2013). Raw soy sauce is, therefore, also characterized by a milder aroma and lighter, less salty flavor (Kaneko, Kumazawa \& Nishimura, 2013; Obata \& Kuwagaki, 2013). In addition, enzymatic activities, such as those of $\alpha$-amylase and proteases, remain intact (Motai, Hayashi, Ishiyama \& Sonehara, 1983; Ando, Kitao, Hatanaka, Sakaue \& Obata, 2020). This is thought to impact the properties of 
various foods prepared with soy sauce.

For example, when raw soy sauce is used as an ingredient while cooking rice, rice texture is softer and less brittle (Ando et al., 2020), likely due to starch degradation by $\alpha$-amylase. It is also possible that protease activity releases starch granules and digests granule-associated proteins, causing the free granules to become sticky (Arai, Aoyama \& Watanabe, 1993). An improvement in the taste of rice cooked with raw soy sauce was also observed (Ando et al., 2020). Together, these findings suggest that raw soy sauce may be used to produce food products with different qualities to those manufactured using heat-treated soy sauce.

A traditional Japanese dish known as "zuke" is prepared by marinating tuna in soy sauce. Marinating time typically varies from 5 to 10 min depending on tuna condition and consumer preference, but may be as long as 60 min (Tsuji, 1980). When mackerel or tuna were marinated in miso or soy sauce for nine days, the fish meat protein became low molecular weight, while the high molecular weight component of tuna tended to remain. (Kawamura \& Yamazaki, 1996). Furthermore, soy sauce not only increased the saltiness of grilled swordfish, but also contributed favorably to caramelization and the aroma produced during cooking, both of which enhanced flavor (Shibata, Watanabe, Imamura, Obata \& Yasuhara, 2015). However, both of these studies used conventional heat-treated soy sauce. No studies have yet investigated the impact of raw soy sauce enzymatic activity on the quality of fish-containing foods. Therefore, the present study investigated the impact of raw soy sauce on the quality of the prepared "zuke," including properties such as texture (associated with the degree of muscle protein degradation).

\section{Material Studied}

Yellowfin tuna (Thunnus albacares; Pacific Ocean, Shizuoka, Japan), soy sauce made from defatted soybeans (Kikkoman Co., Tokyo, Japan), and mirin (Hon Mirin; Kikkoman Co.) were purchased from a retail store. Yellowfin tuna was stored at $4{ }^{\circ} \mathrm{C}$ until use. Both heat-treated (hereafter referred to as heated) and raw soy sauces were purchased, and were frozen in aliquots at $-18{ }^{\circ} \mathrm{C}$ until use. The required volume of soy sauce was gradually brought to room temperature before each use. Manufacturer-provided salt equivalents (converted from sodium) were $13.5 \mathrm{~g} / 100 \mathrm{~g}$ and $13.3 \mathrm{~g} / 100 \mathrm{~g}$ for heated and raw soy sauces, respectively.

\section{Area Descriptions}

Not applicable.

\section{Methods}

\subsection{Soy Sauce Protease Activity}

Soy sauce protease activity was determined as previously described (Hagiwara \& Akahori, 1956). Briefly, soy sauce was placed into a 2-mL dialysis membrane (Ieda Trading Co., Tokyo, Japan) and left in distilled water overnight with constant agitation. Dialysate was diluted 10-fold using $0.2 \mathrm{M}$ phosphate buffer (pH 7.0). A $1 \mathrm{~mL}$ aliquot was mixed with $1 \mathrm{~mL}$ of $2 \%(\mathrm{w} / \mathrm{v})$ casein solution (Hammarsten; Merck, Kenilworth, NJ, USA) as the protease substrate. The reaction proceeded for $10 \mathrm{~min}$ at $30{ }^{\circ} \mathrm{C}$ and was terminated using $4 \mathrm{~mL}$ of $5 \%$ trichloroacetic acid (Wako, Tokyo, Japan). The solution was filtered using No. 5C filter paper $(9 \mathrm{~cm}$ diameter) (Advantec, Tokyo, Japan) and $0.5 \mathrm{~mL}$ of filtrate was combined with $2.5 \mathrm{~mL}$ of $0.4 \mathrm{M}$ sodium carbonate solution (Wako, Tokyo, Japan) and $0.5 \mathrm{~mL}$ of phenol reagent (Wako, Tokyo, Japan). The solution was incubated for 30 $\mathrm{min}$ at $30^{\circ} \mathrm{C}$ before measuring the optical density (OD) at $660 \mathrm{~nm}$ by U-1900 Spectrophotometer (Hitachi, Tokyo, Japan) to calculate protease activity. One protease unit (PU) was defined as the enzyme titer able to produce tyrosine equivalent to $1 \mu \mathrm{g}$ of protein per minute of reaction time. In this experiment, $\mathrm{OD}_{660}=1.00$ was equivalent to $113 \mu \mathrm{g}$ of tyrosine.

\subsection{Tuna Preparation}

Using a sterile knife, cutting board, and gloves, Tuna meat was cut into $5 \times 2 \times 1 \mathrm{~cm}$ slices; $10 \mathrm{~cm}^{3}$. Slices were immersed in a seasoning solution containing a total of $6 \mathrm{~mL}$ with the ratio 1:1 for either heated or raw soy sauce and cooled microwaved mirin, for $0,10,35$, or $60 \mathrm{~min}$ at $4{ }^{\circ} \mathrm{C}$. Mirin was microwaved $(600 \mathrm{~W}, 10 \mathrm{~s})$ to remove alcohol. Unmarinated tuna slices ( 0 min immersion interval) were used as the control. The slices were stored in polyethylene bags where the entire slices covered with the sauce and marinated for the prescribed time. Slices were freeze-dried in a vacuum-assisted VO-250 freeze dryer (TAITEC Corporation, Saitama, Japan), finely ground in an IFM-620DG mill (Iwatani Corporation, Osaka, Japan), and stored in polyethylene bags (Asahi Kasei Home Products Corporation, Tokyo, Japan) at $-80{ }^{\circ} \mathrm{C}$.

\subsection{Change in Tuna Mass}

Following the marinating interval, tuna slices were gently blotted on both sides $(1 \mathrm{~s} \mathrm{each})$ using a paper towel 
(Nippon Paper Cresia Corporation). Slices were weighed, and proportion of mass lost was calculated as follows:

Mass lost $(\%)=100-$ [post-immersion mass $(\mathrm{g}) /$ pre-immersion mass $(\mathrm{g}) \times 100]$

\subsection{Change in Tuna Salt Content}

An aliquot $(100 \mathrm{mg})$ of each powdered tuna slice was suspended in $2 \mathrm{~mL}$ distilled water and centrifuged at $13500 \times \mathrm{g}$ for $10 \mathrm{~min}$ in a refrigerated $\left(4{ }^{\circ} \mathrm{C}\right)$ microcentrifuge (3700; Kubota Corporation, Tokyo, Japan). Supernatant salinity (\%) was measured using an electrical conductivity-based digital salinometer (ES-421; ATAGO, Tokyo, Japan).

\subsection{Change in Tuna Surface Elemental Composition}

Elemental composition analysis was performed to determine the depth of salt $(\mathrm{NaCl})$ penetration into tuna slices.

Following the immersion interval, tuna cut sections of each were obtained using a sterile knife and mounted on a 15 mm-diameter sample holder lined with a conductive carbon sheet (Nissin EM, Tokyo, Japan). Sections were freeze-dried and subjected to scanning electron microscopy (SEM) (TM-3030; HITACHI, Tokyo, Japan) and elemental analyzer (Swift ED3000; Oxford Instruments, Abingdon, Oxfordshire, UK). The observation/analysis area was set at a depth of $3 \mathrm{~mm}$ from the sample surface in order to investigate the degree of penetration.

\subsection{Soy Sauce Color and Change in Tuna Color}

A colorimeter (Z-300A; Nippon Denshoku Industries Co., Ltd., Tokyo, Japan) was used to measure L*(lightness), $\mathrm{a}^{*}(\mathrm{Hue})$, and $\mathrm{b}^{*}$ (saturation) values of soy sauce and of the blotted tuna slice surfaces. A total of three measurements were taken per soy sauce type, and four sites per tuna slice (two sites per side) were surveyed, including three readings per site.

\subsection{Change in Tuna Physical Properties}

\subsubsection{Rupture Load}

A 3 mm-diameter cylindrical plunger (No.3; Yamaden, Tokyo, Japan) was attached to a creepmeter (RE2-33005S; Yamaden, Tokyo, Japan). The plunger was pressed into the tuna slice in a direction parallel to that of the muscle cells at a speed of $1 \mathrm{~mm} / \mathrm{s}$ until the muscle cells ruptured. The maximum value thus obtained was defined as the rupture load (Ando, Ando, Tsukamasa, Makinodan, \& Miyoshi, 1999). Measurement strain rate was set to $98.00 \%$.

\subsubsection{Surface Wetness}

Surface wetness was measured using friction as a proxy, as previously described (Osuga, Iwasaki, Takahashi, \& Ogoshi, 2013). Briefly, a sample container was lined with water-resistant No. 500 sandpaper (Sankyo-Rikagaku Corporation, Okegawa, Saitama) in order to prevent sample movement, and the tuna slice was placed on it. A square plunger with surface area of $250 \mathrm{~mm}^{2}(250 \times 10 \mathrm{~mm})$ was placed in contact with the surface of the tuna slice, attached to a creepmeter (RE-33005B (XZ); Yamaden), and moved over the tuna surface. Measurement conditions were as follows: vertical load of $0.1 \mathrm{~N}$, sliding distance of $20 \mathrm{~mm}$, sliding speed of $1 \mathrm{~mm} / \mathrm{s}$, and measurement time of $20 \mathrm{~s}$. In this manner, initial friction (peak resistance at onset of slide) and average friction (average resistance across sliding distance during measurement time) were calculated; the higher these values, the lower the degree of surface wetness.

\subsection{Change in Tuna Protein Composition}

Protein composition was examined via sodium dodecyl sulfate (SDS)-polyacrylamide gel electrophoresis (PAGE) (Laemmli, 1970). Briefly, approximately $30 \mathrm{mg}$ of powdered tuna was placed in a microtube, and ten times the volume of SDS sample preparation buffer (0.05 M Tris-HCl, $\mathrm{pH} 6.8$, containing $2 \%$ SDS, $10 \%$ glycerol, and $6 \%$ $\beta$-mercaptoethanol) was added. The microtube was boiled in a water bath for $5 \mathrm{~min}$ to achieve sample resuspension and dissolution. Precast 5-20\% polyacrylamide gradient gels (Mini-PROTEAN® TGXTM Gels; Bio-Rad Laboratories, Inc., Hercules, CA, USA) were placed in a gel apparatus and covered with running buffer $(25 \mathrm{mmol} / \mathrm{l}$ Tris, $192 \mathrm{mmol} / \mathrm{l}$ Glycine, $0.1 \%(\mathrm{w} / \mathrm{v}) \mathrm{SDS}$ ). Aliquots $(5 \mu \mathrm{L})$ of resuspended sample solution were placed in each well of the gel. Aliquots $(5 \mu \mathrm{L})$ of the molecular weight marker (Blue Prestained Protein Standard, Broad Range (11-190 kDa); Bio-Rad Laboratories, Inc.) were placed in the two flanking wells. Protein separation proceeded in constant current mode $(20 \mathrm{~mA})$. Thereafter, protein bands were visualized by immersing the gel for approximately $1 \mathrm{~h}$ in the staining solution: 1\% CBB-R250 (ICN Biomedicals Inc., Costa Mesa, CA, USA) dissolved in 50\% methanol (Wako, Tokyo, Japan), 5\% acetic acid (Wako, Tokyo, Japan). The gel was rinsed using distilled water and the background was decolorized via immersion in $7 \%$ acetic acid. 


\subsection{Subjective Evaluation of Tuna Sensory Properties}

Sensory properties of tuna slices marinated for 10 or $60 \mathrm{~min}$ were evaluated by an independent panel (described below) blinded to soy sauce type and marinating interval. One slice from each marinating group was presented on a $10 \mathrm{~cm}$-diameter white plastic plate. A nine-point scoring system for seven properties (appearance, aroma, wetness, softness, saltiness, umami, and overall evaluation) was implemented. Appearance and aroma were evaluated prior to tasting. For appearance, umami, and overall evaluation, a higher score indicated a higher preference. For aroma, wetness, softness, and saltiness, a higher score corresponded directly with a higher degree of intensity of each property. In addition, a column was provided for freeform comments and impressions.

The panel consisted of 19 female students ( $21.5 \pm 0.8$ years old) from the Department of Health and Nutrition, Faculty of Health and Nutrition, Osaka Shoin Women's University. Panel members had prior experience in sensory property evaluation, provided informed consent, and underwent an allergy survey. Study protocols were approved by the Research Plan Review Board and Bioethics Subcommittee of Osaka Shoin Women's University (approval number 28-09) and the study proceeded in accordance with the principles of the Declaration of Helsinki.

\subsection{Statistical Analysis}

Each experiment was performed at least in sextuplicate and up to octuplicate. All data (regarding soy sauce-induced changes in tuna weight, salt content, elemental composition, color, and physical properties) are presented as the mean \pm standard error. All statistical analyses were performed using Office365 (Microsoft Corporation, Redmond, WA, USA) in conjunction with a statistical add-in (BellCurve version 3.20; Social Survey Research Information Corporation, Tokyo, Japan). Groups within each experiment were compared using a one-way analysis of variance, and multiple comparisons were performed using the Tukey test. Student's t-test was used to compare between-group sensory evaluation data. Differences were considered statistically significant at $\mathrm{p}<0.05$.

\section{Results}

\subsection{Soy Sauce Protease Activity}

Protease activity was $0 \mathrm{U} / \mathrm{mL}$ for heated and $25.7 \mathrm{U} / \mathrm{mL}$ for raw soy sauce.

\subsection{Loss of Tuna Mass}

Compared to a marinating interval of $10 \mathrm{~min}$, longer intervals of 35 and 60 min resulted in significant tuna mass loss in both the heated and raw soy sauce-marinated groups (Table 1). However, there was no significant difference in induced mass loss between the heated and raw soy sauce-marinated groups.

Table 1. Proportion of mass lost $(\%)$ as a function of marinating interval

\begin{tabular}{llll}
\hline Marinating interval (min) & 10 & 35 & 60 \\
\hline Heated soy sauce & $2.0 \pm 0.0^{\mathrm{a}}$ & $5.2 \pm 0.7^{\mathrm{b}}$ & $5.6 \pm 0.8^{\mathrm{b}}$ \\
Raw soy sauce & $2.0 \pm 0.1^{\mathrm{a}}$ & $5.0 \pm 0.5^{\mathrm{b}}$ & $6.0 \pm 0.7^{\mathrm{b}}$ \\
\hline
\end{tabular}

Values represents the means \pm standard error $(n=6)$.

Different letters show significant difference $(\mathrm{p}<0.05)$ in mean values by Tukey test.

\subsection{Increase in Tuna Salt Content}

Compared to control tuna (unmarinated), salt content (\%) increased significantly and time-dependently in tuna marinated in both heated and raw soy sauce (Table 2). However, there was no significant difference with respect to the increase in the induced salt content (\%) between the heated and raw soy sauce-marinated groups.

Table 2. Salt content $(\%)$ increase as a function of marinating interval

\begin{tabular}{lllll}
\hline Marinating interval (min) & 0 & 10 & 35 & 60 \\
\hline Heated soy sauce & $0.15 \pm 0.00^{\mathrm{a}}$ & $0.36 \pm 0.01^{\mathrm{b}}$ & $0.38 \pm 0.01^{\mathrm{c}}$ & $0.43 \pm 0.02^{\mathrm{d}}$ \\
Raw soy sauce & & $0.35 \pm 0.01^{\mathrm{b}}$ & $0.38 \pm 0.00^{\mathrm{c}}$ & $0.41 \pm 0.01^{\mathrm{d}}$ \\
\hline
\end{tabular}

Values represents the means \pm standard error $(n=6)$.

Different letters show significant difference $(\mathrm{p}<0.05)$ in mean values by Tukey test. 


\subsection{Increase in Tuna Surface $\mathrm{NaCl}$}

Compared to control tuna (unmarinated) and marinating intervals of 10 and $35 \mathrm{~min}$, a longer interval of $60 \mathrm{~min}$ produced significant increases in $\mathrm{Na}$ and $\mathrm{Cl}$ content, but only in the raw soy sauce-marinated group (Table 3). This indicates greater penetration of $\mathrm{NaCl}$ into tuna during exposure to raw soy sauce.

Table 3. $\mathrm{Na}$ and $\mathrm{Cl}$ content (\% by mass) as a function of marinating interval and soy sauce type

\begin{tabular}{lllllllll}
\hline & \multicolumn{9}{c}{$\begin{array}{c}c \\
\text { Na }\end{array}$} & & & \multicolumn{3}{c}{ Cl } \\
\hline $\begin{array}{l}\text { Marinating } \\
\text { interval (min) } \\
\text { heated }\end{array}$ & 0 & 10 & 35 & 60 & 0 & 10 & 35 & 60 \\
$\begin{array}{l}\text { Soy sauce } \\
\text { raw }\end{array}$ & n.d. & $0.01 \pm 0.00^{\mathrm{a}}$ & $0.13 \pm 0.12^{\mathrm{b}}$ & $0.26 \pm 0.08^{\mathrm{c}}$ & n.d. & $0.01 \pm 0.00^{\mathrm{a}}$ & $0.10 \pm 0.14^{\mathrm{b}}$ & $0.22 \pm 0.07^{\mathrm{c}}$ \\
Soy sauce & n.d. & $0.02 \pm 0.05^{\mathrm{a}}$ & $0.23 \pm 0.05^{\mathrm{bc}}$ & $0.46 \pm 0.09^{\mathrm{d}}$ & n.d. & $0.03 \pm 0.03^{\mathrm{a}}$ & $0.07 \pm 0.02^{\mathrm{ab}}$ & $0.32 \pm 0.09^{\mathrm{d}}$ \\
\hline
\end{tabular}

Values represents the means \pm standard error $(n=6)$, Abbreviations: $n . d$. not detected.

Different letters show significant difference $(\mathrm{p}<0.05)$ in mean values by Tukey test.

\subsection{Tuna Physical Properties}

\subsubsection{Decrease in Rupture Load}

Compared to control tuna (unmarinated), heated and raw soy sauces initially induced equivalent decreases in rupture load (Figure 1). In addition, beyond the shortest marinating interval (10 $\mathrm{min})$, a significantly greater decrease in rupture load was observed in the raw soy sauce-marinated group. This indicates greater softening of tuna during exposure to raw soy sauce for longer intervals.

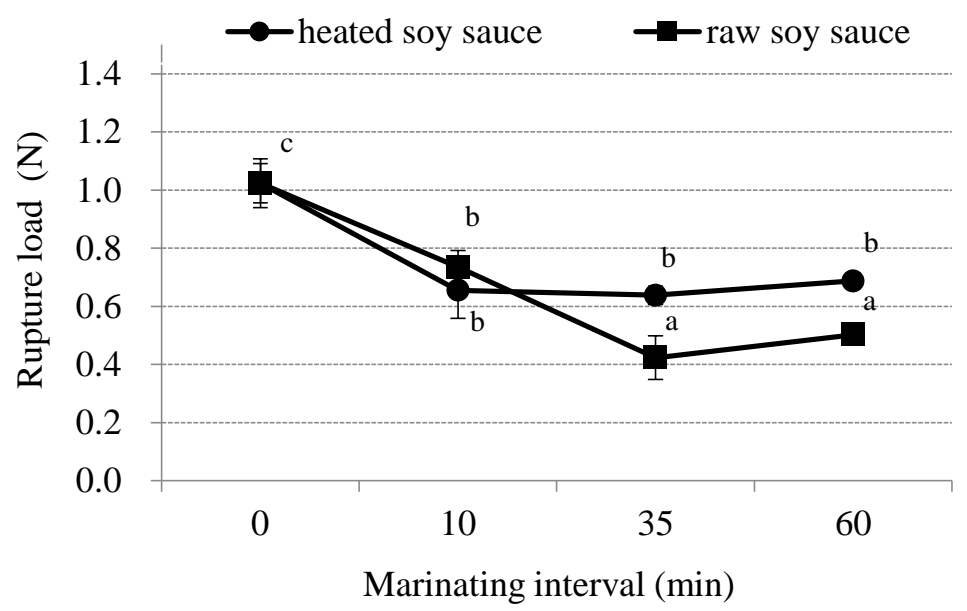

Figure 1. Change in rupture load as a function of marinating interval and soy sauce type

Lines in circle mark are presented using heated soy sauce and lines in square mark are presented using raw soy sauce, respectively. Statistical analysis was performed using the multiple comparison test, Tukey, with one-way ANOVA to compare differences in means at $p<0.05(\mathrm{n}=6)$. Different letters in each evaluation item show significant differences in each same marinating interval.

\subsubsection{Differential Change in Surface Wetness Creep Meter}

In the raw soy sauce-marinated group: compared to control tuna (unmarinated), initial friction decreased significantly after $10 \mathrm{~min}$ of marinating, but longer marinating intervals did not produce any additional significant change in initial friction (Figure 2). In the heated soy sauce-marinated group: compared to control tuna (unmarinated) and a marinating interval of $10 \mathrm{~min}$, initial friction increased significantly after $35 \mathrm{~min}$ of marinating, but a longer marinating interval did not produce any additional significant change in friction. For all marinating intervals, initial friction in the raw soy sauce-marinated group was significantly lower than that in the heated soy sauce-marinated group. This indicates a more slippery surface in the raw soy sauce-marinated group. 


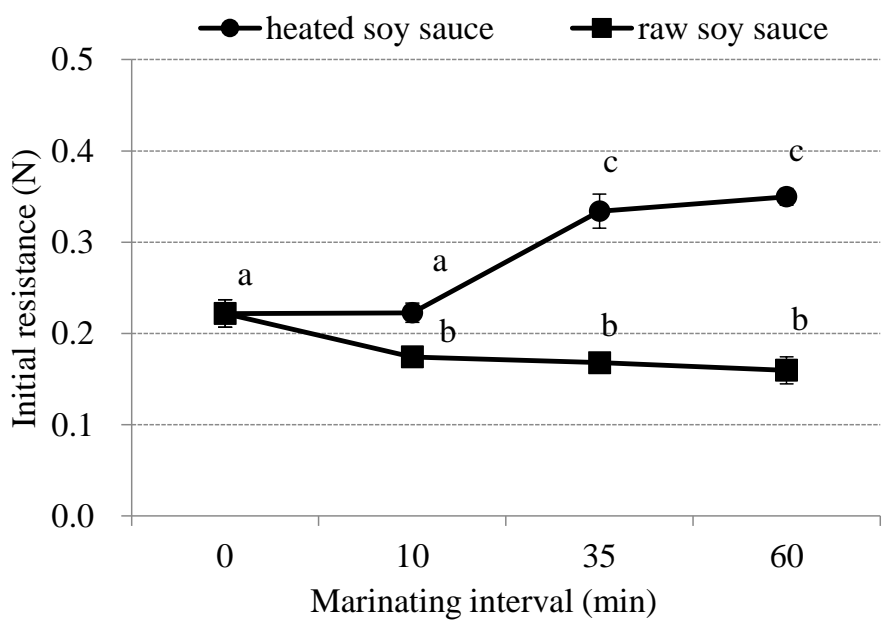

Figure 2. Change in initial resistance as a function of marinating interval and soy sauce type

Lines in circle mark are presented using heated soy sauce and lines in square mark are presented using raw soy sauce, respectively. Statistical analysis was performed using the multiple comparison test, Tukey, with one-way ANOVA to compare differences in means at $p<0.05(\mathrm{n}=6)$. Different letters in each evaluation item show significant differences in each same marinating interval.

In both the heated and raw soy sauce-marinated groups, average friction increased (Figure 3). This increase was already significant after a marinating interval of $10 \mathrm{~min}$ in the heated soy sauce-marinated group, and became significant after a marinating interval of $35 \mathrm{~min}$ in the raw soy sauce-marinated group. This suggests that while between-group differences in slipperiness were captured at slide onset, dehydration-induced surface firming prevailed in both groups during the subsequent slide distance.

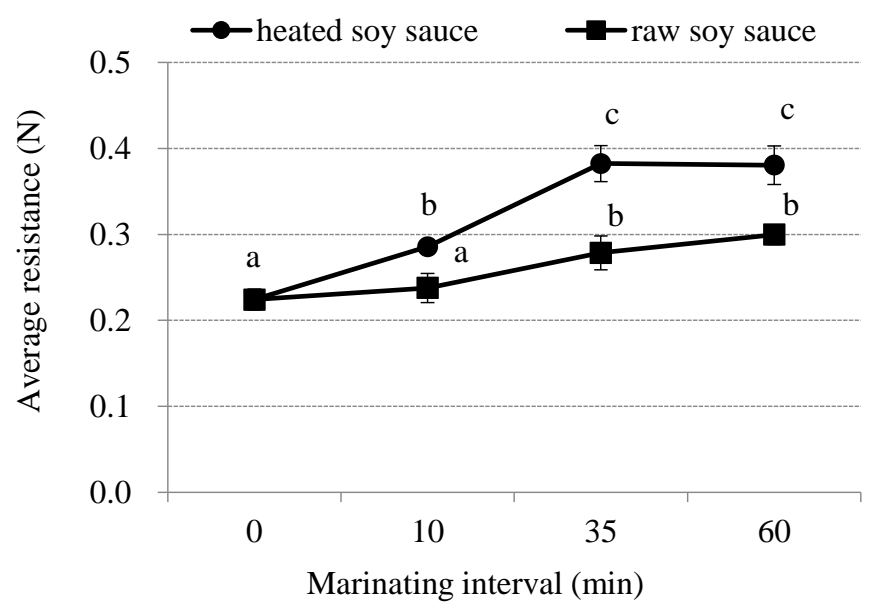

Figure 3. Change in average resistance as a function of marinating interval and soy sauce type

Lines in circle mark are presented using heated soy sauce and lines in square mark are presented using raw soy sauce, respectively. Statistical analysis was performed using the multiple comparison test, Tukey, with one-way ANOVA to compare differences in means at $p<0.05(\mathrm{n}=6)$. Different letters in each evaluation item show significant differences in each same marinating interval.

\subsection{Soy Sauce Color and Change in Tuna Color}

In both the heated and raw soy sauce-marinated groups: compared to control tuna (unmarinated), a 35-min marinating interval significantly decreased $\mathrm{L}^{*}$ values (indicating a lighter/darker color) (Table 4). However, there was no significant difference between the heated and raw soy sauce-marinated groups. In the raw soy sauce-marinated group: compared to control tuna (unmarinated), a 60-min marinating interval significantly increased $\mathrm{a}^{*}$ values (indicating reddening). Also in the raw soy sauce-marinated group: compared to control tuna (unmarinated), a 35-min marinating interval significantly increased $b^{*}$ values (indicating brighter color). In the 
heated soy sauce-marinated group: compared to control tuna (unmarinated), a 60-min marinating interval significantly increased $b^{*}$ values. However, there was no significant difference between the heated and raw soy sauce-marinated groups. From the results of a* values, it was clear that tuna marinated in raw soy sauce had a reddish color when soaked for 60-min. Reddish color is a characteristic of tuna sashimi, and it is expected that tuna dipped in raw soy sauce will have a stronger reddish color and be more palatable.

Table 4. Color change as a function of marinating interval and soy sauce type

\begin{tabular}{|c|c|c|c|c|c|c|c|c|c|}
\hline & $\mathrm{L}^{*}$ & & & $a^{*}$ & & & $b^{*}$ & & \\
\hline & blank & $\begin{array}{l}\text { heated } \\
\text { soy sauce }\end{array}$ & $\begin{array}{l}\text { raw } \\
\text { soy sauce }\end{array}$ & blank & $\begin{array}{l}\text { heated } \\
\text { soy sauce }\end{array}$ & $\begin{array}{l}\text { raw } \\
\text { soy sauce }\end{array}$ & blank & $\begin{array}{l}\text { heated } \\
\text { soy sauce }\end{array}$ & $\begin{array}{l}\text { raw } \\
\text { soy sauce }\end{array}$ \\
\hline $0 \min$ & $41.7 \pm 1.3^{\mathrm{a}}$ & - & - & $22.5 \pm 0.5^{\mathrm{a}}$ & - & - & $19.7 \pm 0.3^{\mathrm{a}}$ & - & - \\
\hline $10 \mathrm{~min}$ & - & $38.4 \pm 0.6^{\mathrm{abc}}$ & $39.5 \pm 0.7^{\mathrm{ab}}$ & - & $23.3 \pm 0.4^{\mathrm{ab}}$ & $23.8 \pm 1.1^{\mathrm{ab}}$ & - & $21.1 \pm 1.1^{\mathrm{a}}$ & $23.0 \pm 0.9^{\mathrm{ab}}$ \\
\hline $35 \mathrm{~min}$ & - & $37.0 \pm 0.5^{\mathrm{bc}}$ & $37.5 \pm 1.0^{b c}$ & - & $23.3 \pm 1.0^{\mathrm{ab}}$ & $25.1 \pm 0.6^{\mathrm{ab}}$ & - & $22.1 \pm 1.0^{\mathrm{ab}}$ & $24.2 \pm 0.4^{\mathrm{b}}$ \\
\hline $60 \mathrm{~min}$ & - & $35.4 \pm 1.0^{c}$ & $37.2 \pm 0.8^{\mathrm{bc}}$ & - & $23.6 \pm 0.5^{\mathrm{a}}$ & $26.3 \pm 0.7^{\mathrm{b}}$ & - & $23.9 \pm 0.8^{\mathrm{b}}$ & $24.5 \pm 0.8^{\mathrm{b}}$ \\
\hline
\end{tabular}

Values represents the means \pm standard error $(n=8)$.

Different letters show significant difference $(\mathrm{p}<0.05)$ in the same color in mean values by Tukey test.

Color measurement values for heated soy sauce $\left(\mathrm{L}^{*}=16.9 \pm 0.1, \mathrm{a}^{*}=45.0 \pm 0.1\right.$, and $\left.\mathrm{b}^{*} 28.3 \pm 0.0\right)$ were significantly lower than those for raw soy sauce $\left(L^{*}=23.5 \pm 0.1, a^{*}=50.5 \pm 0.2\right.$, and $\left.b^{*}=39.1 \pm 0.1\right)$, indicating that although both soy sauce types are reddish in color, raw soy sauce color is lighter and brighter (Table 5).

Table 5. Color tone of heated and raw soy sauce

\begin{tabular}{llll}
\hline & $\mathrm{L}^{*}$ & $\mathrm{a}^{*}$ & $\mathrm{~b}^{*}$ \\
\hline Heated soy sauce & $16.9 \pm 0.1^{\mathrm{a}}$ & $45.0 \pm 0.1^{\mathrm{a}}$ & $28.3 \pm 0.0^{\mathrm{a}}$ \\
Raw soy sauce & $23.3 \pm 0.1^{\mathrm{b}}$ & $50.5 \pm 0.2^{\mathrm{b}}$ & $39.1 \pm 0.1^{\mathrm{b}}$ \\
\hline
\end{tabular}

Values represents the means \pm standard error $(\mathrm{n}=8)$.

Different letters show significant difference $(\mathrm{p}<0.05)$ in the same color in mean values by Tukey test.

\subsection{Differential Change in Tuna Protein Composition}

For all marinating intervals, the protein band corresponding to myosin heavy chain (MHC) was smaller in the raw soy sauce-marinated group than in the heated soy sauce-marinated group (especially around $200 \mathrm{kDa}$ ) (Figure 4), indicating degradation of MHC (and likely various other proteins). 


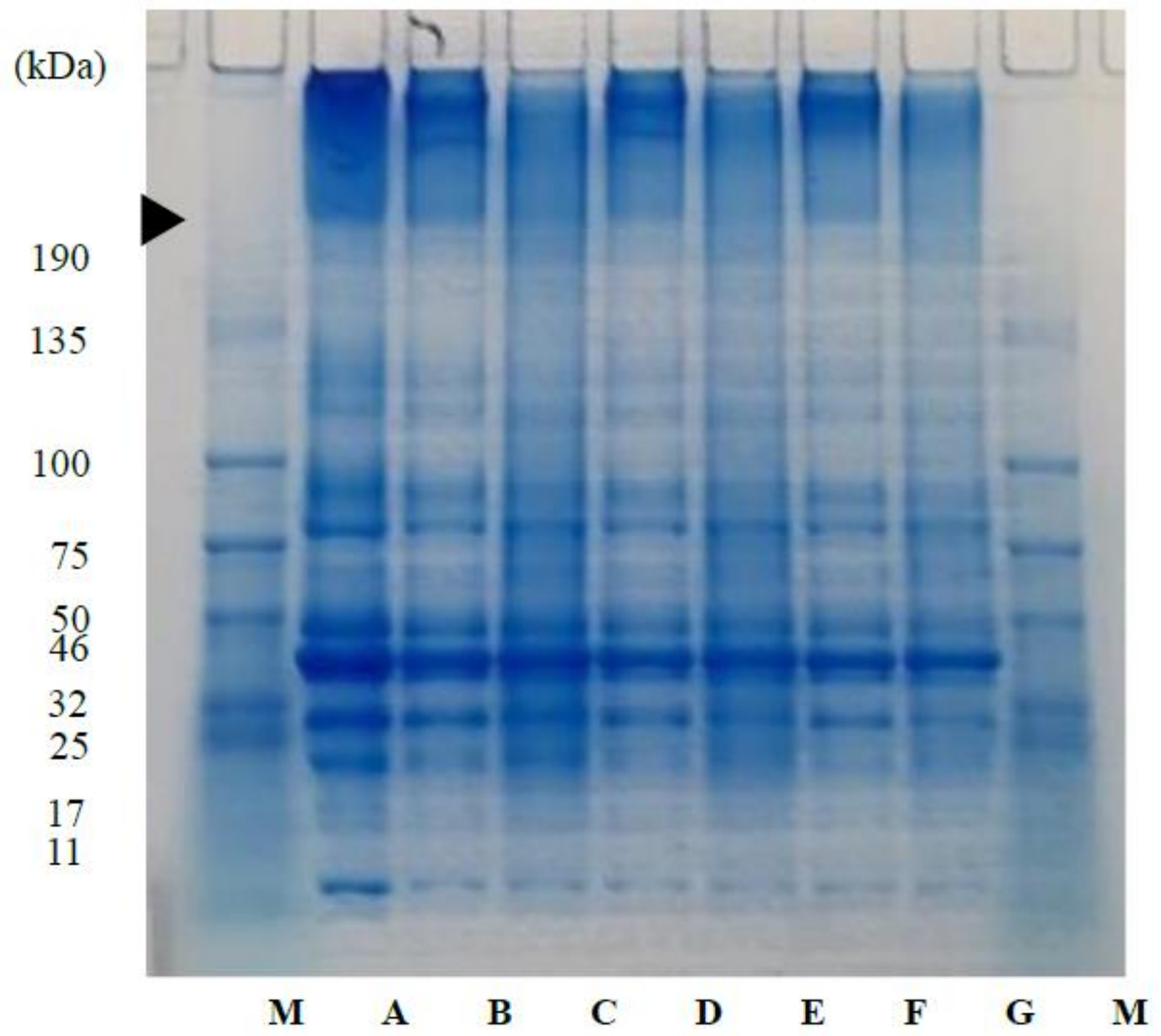

Figure 4. Polyacrylamide gradient (5-20\%) gel electrophoretic pattern of tuna proteins as a function of marinating interval and soy sauce type. The arrowhead indicates myosin heavy chain protein

Lanes: M molecular weight (MW) marker, A control, B heated soy sauce (10 min), C raw soy sauce (10 min), D heated soy sauce (35 min), E raw soy sauce (35 min), F heated soy sauce $(60 \mathrm{~min}), \mathrm{G}$ raw soy sauce $(60 \mathrm{~min})$, Arrow head MHC

\subsection{Subjective Evaluation of Tuna Sensory Properties}

Regarding appearance, raw soy sauce-marinated tuna scored significantly higher than heated soy sauce-marinated tuna (Figure 5), and frequent freeform comment mentions of "shiny" associated with the former suggested that this preference was likely attributable to increased surface wetness (rather than differences in color). Regarding aroma, no significant difference in score was noted between heated and raw soy sauce-marinated tuna. Regarding surface wetness, raw soy sauce-marinated tuna scored significantly higher than heated soy sauce-marinated tuna. However, a difference in respondent preference was revealed in freeform comments: while most respondents evaluated raw soy sauce-marinated tuna favorably (including terms such as "good texture" and "smooth"), some mentioned that "heated soy sauce [-marinated tuna] is less wet and preferable". Regarding softness, raw soy sauce-marinated tuna tended to score more highly than heated soy sauce-marinated tuna, but the difference was not statistically significant. Regarding saltiness, heated soy sauce-marinated tuna tended to score as saltier than raw soy sauce-marinated tuna, although this difference only became statistically significant after the longer marinating interval of $60 \mathrm{~min}$. Most respondents rated raw soy sauce-marinated tuna saltiness as "just right," while rating heated soy sauce-marinated tuna saltiness as "salty" or "too strong." Regarding umami flavor, raw soy sauce-marinated tuna tended to score more highly than heated soy sauce-marinated tuna, although this difference only became statistically significant after the longer marinating interval of $60 \mathrm{~min}$. Regarding overall taste preference (deliciousness), raw soy sauce-marinated tuna scored significantly higher than heated soy sauce-marinated tuna (following the 60-min marinating interval). Multiple respondents commented that raw soy sauce-marinated tuna tasted "mellow" and "had the original taste of tuna." 
(a)

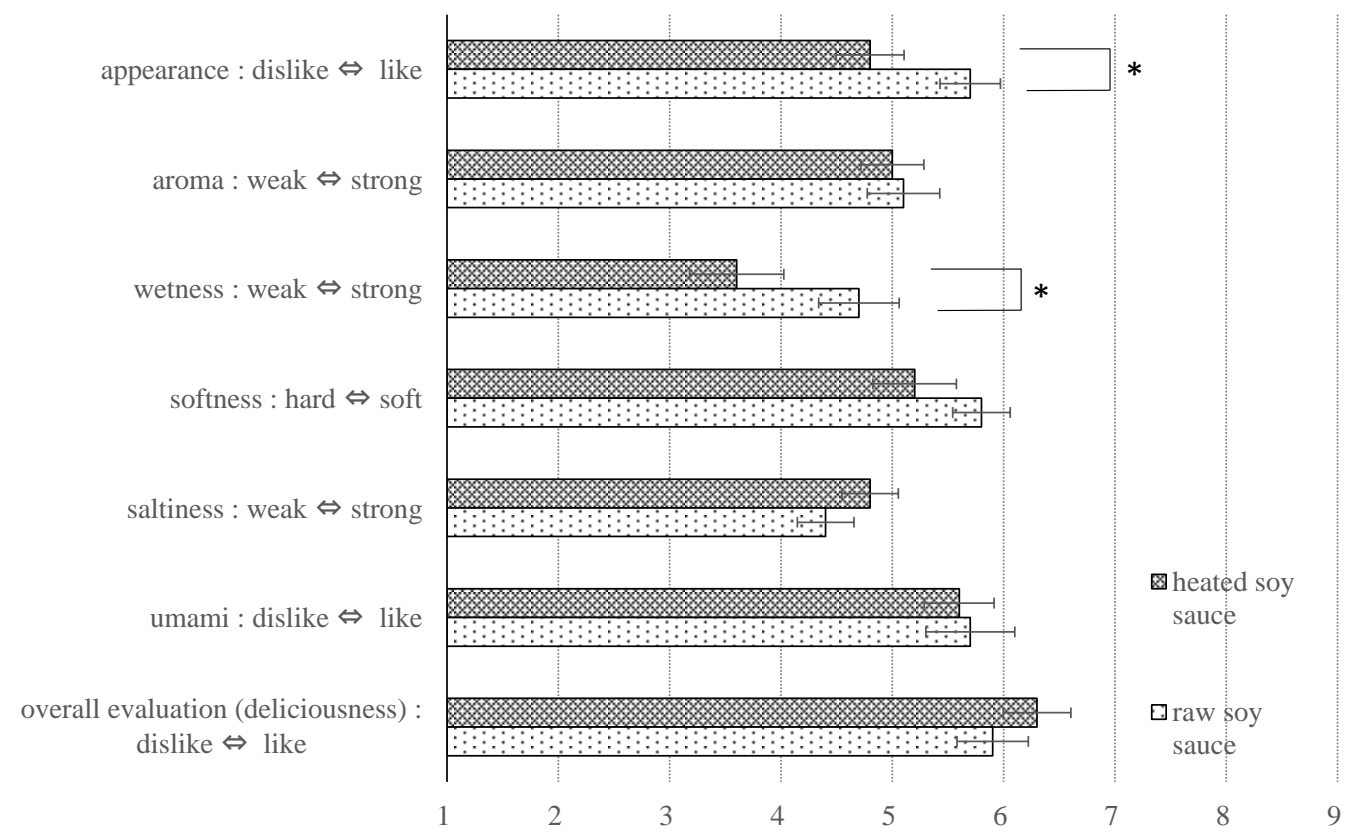

(b)

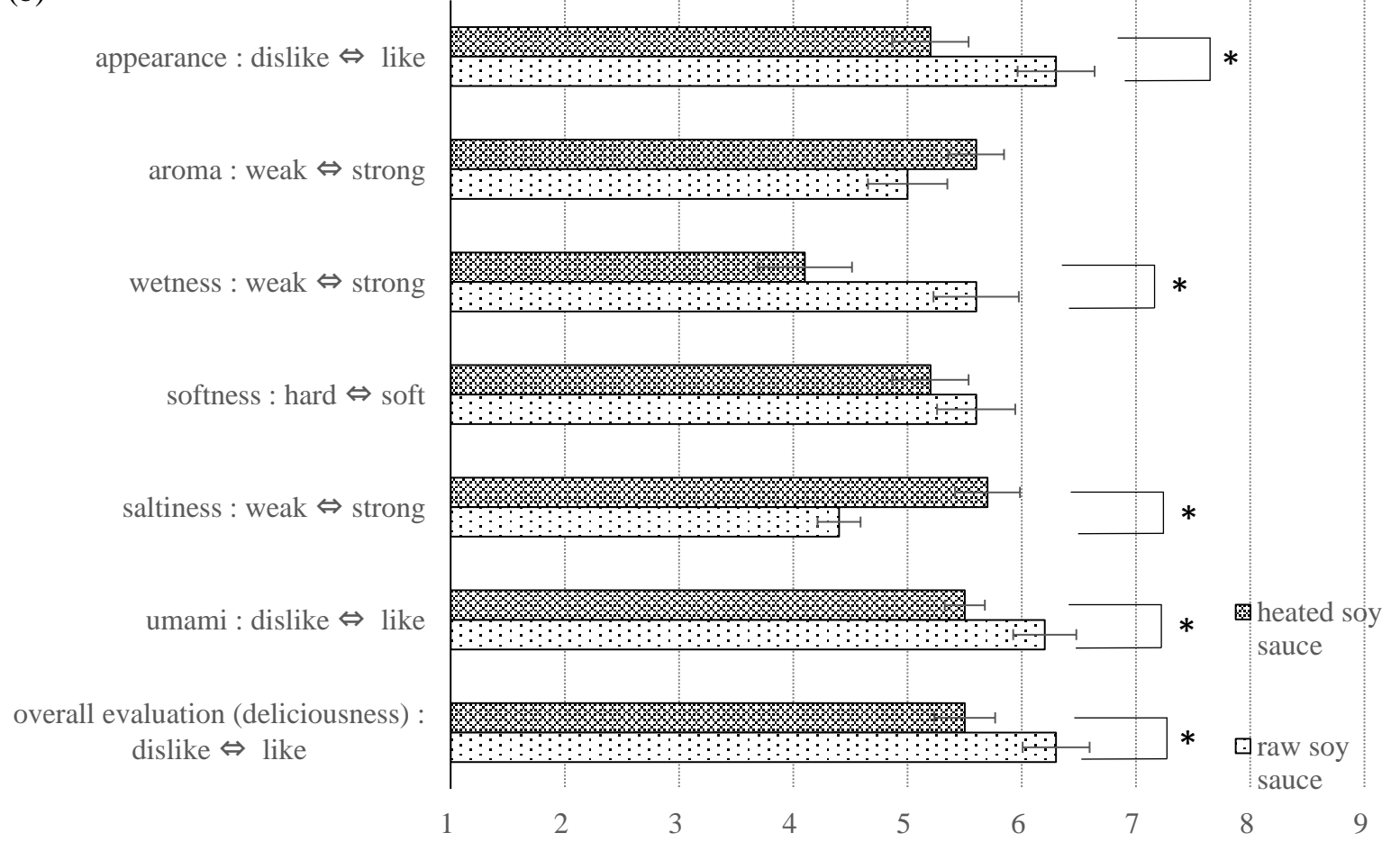

Figure 5. Sensory property scores of tuna marinated for $10 \mathrm{~min}$ (a) and $60 \mathrm{~min}$ (b) as a function of soy sauce type

Cross mark bar are presented using heated soy sauce and dot mark bar are presented using raw soy sauce, respectively. Data are expressed as means \pm standard error $(n=19)$. Statistical analysis was performed using the t-test $(p<0.05)$. A symbol $(*)$ show significant differences.

\section{Discussion}

Modern food diversification dictates that the food industry should investigate the impact of soy sauce on food properties in order to optimize soy sauce use and identify novel soy sauce applications. While soy sauce is often 
used as a seasoning or condiment in association with red meat dishes such as steaks and hamburgers, it also has the potential to contribute to the development of new seafood dishes unique to each country (Diez-Simon, Eischelsheim, Mumm \& Hall, 2020). Raw soy sauce may be of particular utility in such applications.

Koji mold is used during the early stages of soy sauce preparation, and the activity of mold-derived enzymes (such as amylase and proteases) are preserved by avoiding heat treatment (Diez-Simon et al., 2020). Present study findings confirm that heat treatment denatures soy sauce protease enzymes, while filtration-based sterilization preserves protease activity. It has been reported that proteases produced by Aspergillus sojae (used during the production of the raw soy sauce used in the present study) increase meat tenderness (Lee, Kim, Yu, \& Yang, 1975). Consistent with this, the present study demonstrated that longer raw soy sauce marinating intervals produce a greater degree of tuna softening as assessed by rupture load (force load at which the specimen ruptures). This effect was especially pronounced in surface-proximal areas of the specimen, indicating that the tuna surface became softer when exposed to raw soy sauce at longer marinating intervals.

This is likely due to an enzymatic disruption of surface muscle architecture, and indeed the present study demonstrates that tuna exposure to raw soy sauce results in the degradation of MHC (and therefore likely various other proteins). Raw soy sauce produced with the assistance of Aspergillus oryzae contains active serine and aspartic proteases, and it was previously demonstrated that treatment of beef myofibrils with isolated forms of such proteases produces electrophoretic profiles similar to those of beef myofibrils treated with raw soy sauce (Tsuji, Hamano, Kashiyama, \& Fukushima, 1978). Serine proteases, in particular, contribute significantly to myofibrillar protein and collagen degradation (Tsuji et al., 1978). Tuna protein degradation observed in the present study is thus thought to be attributable to the action of raw soy sauce proteases converting tuna myofibril proteins to lower-MW products.

However, no significant difference in subjective "softness" scores between raw soy sauce-marinated and heated soy sauce-marinated tuna was evident. This may be due to the sensation of slipperiness being more pronounced than that of tenderness, or relatedly, that marinating softens largely the tuna surface, while central tuna firmness is preserved and is, therefore, the predominant characteristic perceived during chewing. This may be also due to the salt and acid present in soy sauce that marinade diffuses into the muscle tissues reducing the $\mathrm{pH}$ and resulting the significant hydration of the proteins causing swelling and weakening of the muscles and connective tissues (Goli, Bohuon, Ricci, Trystram \& Collignan, 2011; Kim et al., 2013).

Present study findings also indicate that longer marinating intervals (using either soy sauce type) significantly increase both mass loss and salt content. Indeed, mass loss is directly attributable to salt-induced dehydration, and the heated and raw soy sauces used in the present study had comparable salt contents. Furthermore, the longest marinating interval produces greater depth of $\mathrm{NaCl}$ penetration as assessed by SEM-EDS, but only in raw soy sauce-marinated tuna. This may be due to the action of a cellular architecture-disrupting protease (especially serine protease) and lipase activity facilitating permeability and thus deeper soy sauce penetration. Perhaps surprisingly, salinity measurements did not reflect a significant difference in salt content between the heated and raw soy-sauce marinated tuna. This is likely due to SEM-EDS focusing on the most salt-saturated area of the sample (to a depth of $3 \mathrm{~mm}$ below the surface), while salinity measurements target the entire sample (including the large-volume center) and therefore surface differences in salt content become relatively negligible.

Despite comparable salt contents of the two soy sauce types and no difference in entire-sample salinity, a significant difference in subjective "saltiness" scores between raw soy sauce-marinated and heated soy sauce-marinated tuna was nonetheless noted. Although surface salt content of raw soy sauce-marinated tuna was higher, heated soy sauce-marinated tuna was perceived as saltier. This finding may be attributable to two mechanisms such as the difference of "aroma" in raw and heated soy sauce and the surface wetness of marinated tuna. Although subjective "aroma" scores between raw soy sauce-marinated and heated soy sauce-marinated tuna were equivalent, it is known that the aroma of heated soy sauce is stronger (more consistent with the aroma expected from soy sauce which has strong spicy/burnt, malty, cooked potato-like and caramel-like notes) than that of raw soy sauce (less of malty, cooked potato and spicy/burnt notes), and this may have influenced the perception of saltiness (Kaneko, et. al., 2013). It has been reported that heated soy sauce is perceived as saltier (Kaneko, 2015). Furthermore, increased surface wetness of raw soy sauce-marinated tuna may have modulated the perception of the surface saltiness. This may be caused by the wettability of soy sauce. The wettability of coating solutions on solid surfaces was attributed to a mechanism involving the balance between adhesion and cohesion forces of the solution where adhesive forces arise from the chemical interactions between the coating solution and the surface to be coated by contributing to the wettability of the solution, while cohesive forces arise from the polymer-polymer interactions of the coating solution and exert an opposite effect by causing the liquid to contract (Alcântara, Martins, Sousa, Cerqueira, Silva, Filho \& Souza, 2019). 
Indeed, in the present study, raw soy sauce-marinated tuna had a more slippery surface than heated soy sauce-marinated tuna, as judged by initial friction. It was previously demonstrated that beef collagen and myofibrillar fragmentation indices (solubility) became significantly increased (also contributing to decreased shear force) following a heated soy marinating interval of $72 \mathrm{~h}$ at $4{ }^{\circ} \mathrm{C}$ (Kim et al., 2013). This was attributed to the salt-mediated solubilization of collagen and myofibrillar proteins (Kim et al., 2013; Kim et al., 2014). Proteases (and other active enzymes) in raw soy sauce may further enhance surface slipperiness through partial liquefaction of surface solids. However, the present study also notes that both soy sauce types decreased surface slipperiness, as judged by average friction. This may be due to salt-induced dehydration becoming the predominant feature after initial force application. It has also previously been noted that exposure to soy sauce may initially soften meat, but as exposure intervals become longer, the dehydration effect becomes predominant, thereby gradually firming meat (Kawamura \& Yamazaki, 1996). Firmness of fish marinated in soy sauce for nine days was over twice that of the original firmness (Kawamura \& Yamazaki, 1996). Consistent with these findings, the present study notes that average friction increases as marinating intervals become longer (independent of soy sauce type).

A significant difference in subjective "surface wetness" scores between raw soy sauce-marinated and heated soy sauce-marinated tuna was noted, with the former scoring higher. Even prior to tasting, this perception was evident, and significantly modulated "appearance" scores: despite no difference in measured color between raw and heated soy sauce-marinated tuna following a 10-min marinating interval, respondents significantly preferred the appearance of the former following both marinating intervals. Frequent comment mentions of "shiny" suggested that this preference was likely attributable to increased visual surface wetness of raw soy sauce-marinated tuna. Future studies may investigate this hypothesis using a gloss meter.

While both soy sauce types are reddish in color, heat treatment causes the soy sauce color to darken. The present study confirms that raw soy sauce color was lighter and brighter and demonstrates that exposure to either soy sauce type increased tuna redness, lightness, and brightness (with raw soy sauce more rapidly increasing redness ). It was anticipated that raw soy sauce would have less of an impact on the original (visually pleasing) bright red tuna color than darker heated soy sauce. Indeed, respondents significantly preferred the appearance of raw soy sauce-marinated tuna, but as mentioned above, this was likely due to perceived glossiness, rather than perceived color.

Following a marinating interval of $60 \mathrm{~min}$, respondents significantly preferred the umami flavor and overall deliciousness of the raw soy sauce-marinated tuna.

As is evident from the above observations, two major differential characteristics of raw soy sauce are preserved enzymatic activities and a brighter color. Enzymes can partially degrade biomolecules such as proteins, producing a wide range of effects (e.g., meat tenderization) (Bhat, Morton, Mason \& Bekhit, 2018). The enzyme papain is sometimes injected to break down myofibrillar proteins and tenderize steak (Madhusankha \& Thilakarathna, 2021). As an alternative, marinating raw meat in raw soy sauce may simultaneously season and tenderize it. Furthermore, koji mold enzymes may generate low-MW peptides and sugars with additional desirable bioactivities, such as antioxidant activity (Chen, Muramoto, Yamauchi, \& Nokihara, 1996; Chancharoonpong, Hsieh \& Sheu, 2012). Finally, the bright color of the raw soy sauce can enhance the original colors and appearances of the ingredients themselves (especially when used in conjunction with food preparation methods that do not involve heating), while also enhancing their natural flavor. In this manner, raw soy sauce has the potential to inspire novel culinary preparations and practices, and is expected to find application within a wide variety of future cooking methods and dishes.

\section{Conclusion}

The present study investigated the impact of raw soy sauce, as compared to heated soy sauce, on the properties of tuna "zuke". Raw soy sauce enzymatic activity produced significant surface tenderization and enhanced superficial salt penetration as well as surface glossiness. In a subjective sensory evaluation, respondents preferred raw soy sauce-marinated "zuke" in terms of appearance, saltiness, umami, and overall deliciousness. The authors conclude that raw soy sauce demonstrates potential for wider utility within the global market, and that further study may confirm desirable associated health benefits.

\section{Acknowledgements}

This work was supported by Kikkoman corporation, Tokyo, Japan. We would like to thank Editage (www.editage.jp) for English language editing. 


\section{References}

Alcântara, L. O., Martins, M. E. D. O., Sousa, J. R., Cerqueira, M. Â., Silva, A. L. C., Filho, M. D. S. M. S., \& Souza, B. W. S. (2019). Wettability of edible coatings on Nile tilapia fillets (Oreochromis niloticus). Journal of Food Engineering, 247, 152-159. https://doi.org/10.1016/j.jfoodeng.2018.11.026

Ando, M., Ando, M., Tsukamasa, Y., Makinodan, Y., \& Miyoshi, M. (1999). Muscle firmness and structure of raw and cooked arrow squid mantle as affected by freshness. Journal of Food Science, 64, 659-662. https://doi.org/10.1111/j.1365-2621.1999.tb15105.x

Ando, M., Kitao, S., Hatanaka, Y., Sakaue, R., \& Obata, A. (2020). Characteristics of raw soy sauce for cooked rice. Journal of Cookery Science of Japan, 53, 207-215. https://doi.org/10.11402/cookeryscience.53.207

Arai, E., Aoyama, K., \& Watanabe, M. (1993). Enzymatic improvement of the cooking quality of aged rice: A main mode of protease action. Bioscience, Biotechnology, and Biochemistry, 57, 911-914. https://doi.org/10.1271/bbb.57.911

Bhat, Z. F., Morton, J. D., Mason, S. L., \& Bekhit, A. E. A. (2018) Applied and emerging methods for meat tenderization: A comparative perspective. Comprehensive Reviews in Food Science and Food Safety, 17, 841-859. https://doi.org/10.1111/1541-4337.12356

Chancharoonpong, C., Hsieh, P. C., \& Sheu, S. C. (2012) Enzyme production and growth of Aspergillus oryzae S. on soybean koji fermentation. APCBEE Procedia, 2, 57-61. https://doi.org/10.1016/j.apcbee.2012.06.011

Chen, H. M., Muramoto, K., Yamauchi, F., \& Nokihara, K. (1996). Antioxidant activity of designed peptides based on the antioxidative peptide isolated from digests of a soybean protein. Journal of Agricultural and Food Chemistry, 44(9), 2619-2623. https://doi.org/10.1021/jf950833m

Diez-Simon, C., Eichelsheim, C., Mumm, R., \& Hall, R. D. (2020) Chemical and sensory characteristics of soy sauce: A review. Journal of Agricultural and Food Chemistry, 68, 11612-11630. https://doi.org/10.1021/acs.jafc.0c04274

Goli, T., Bohuon, P., Ricci, J., Trystram, G., \& Collignan, A. (2011). Mass transfer dynamics during the acidic marination of turkey meat. Journal of Food Engineering, 104, 161-168. https://doi.org/10.1016/j.jfoodeng.2010.12.010

Hagiwara, B., \& Akahori, S. (1956). Enzyme research methods. Tokyo, Japan: Asakura Publishing Co., Ltd. pp. 237-240.

Higashi, K. (2008). Fermentation and brewing (1): Guidance on production line and analysis of miso (3rd ed.). Soy sauce, Korin co., Ltd., Tokyo. pp. 61-90.

Kaneko, S. (2015). Studies on key aroma component in raw (unheated) Japanese soy sauce and those changes during heating. Journal of the Brewing Society of Japan, 110(1), 20-25. https://doi.org/10.6013/jbrewsocjapan.110.20

Kaneko, S., Kumazawa, K., \& Nishimura, O. (2013). Studies on the key aroma compounds in raw (unheated) and heated Japanese soy sauce. Journal of Agricultural and Food Chemistry, 61, 3396-3402. https://doi.org/10.1021/jf400353h

Kawamura, F., \& Yamazaki, K. (1996). Change in components and hardness of fish meat cured in miso and soy sauce. Bulletin of the Tokyo College of Domestic Science, 36, 49-52.

Kim, H. W., Choi, Y. S., Choi, J. H., Kim, H. Y., Lee, M. A., Hwang, K. E., ... Kim, C. J. (2013). Tenderization effect of soy sauce on beef M. biceps femoris. Food Chemistry, 139, 597-603. https://doi.org/10.1016/j.foodchem.2013.01.050

Kim, H. W., Hwang, K. E., Song, D. H., Kim, Y. J., Lim, Y. B., Choi, J. H., .. Kim, C. J. (2014). Effects of soy sauce on physicochemical and textural properties of tumbled chicken breast. Poultry Science, 93, 680-686. http://dx.doi.org/ 10.3382/ps.2012-02930

Laemmli, U. K. (1970). Cleavage of structural proteins during the assembly of the head of bacteriophage T4. Nature, 227, 680-685. https://doi.org/10.1038/227680a0

Lee, J. H., Kim, K. W., Yu, J. H., \& Yang, R. (1975). Study on meat tenderizer. Part II. Tenderizing ability of enzyme from Aspergillus oryzae. Korean Journal of Food Science and Technology, 7, 229-239.

Madhusankha, G. D. M. P., \& Thilakarathna, R. C. N. (2021) Meat tenderization mechanism and the impact of plant exogenous proteases: A review. Arabian Journal of Chemistry, 14, 102967. 
https://doi.org/10.1016/j.arabjc.2020.102967

Martins, S. I. F. S., Jongen, W. M. F., \& van Boekel, M. A. J. S. (2000) A review of Maillard reaction in food and implications to kinetic modelling. Trends in Food Science and Technology, 11, 364-373. https://doi.org/10.1016/S0924-2244(01)00022-X

Motai, H., Hayashi, K., Ishiyama, T., \& Sonehara, T. (1983). Role of the main enzyme proteins of koji-mold Aspergillus in sedimentation of coagula in soy sauce during pasteurization. Nippon Nogeikagaku Kaishi, 57, 27-36. https://doi.org/10.1271/nogeikagaku1924.57.27

Nojiro, K., Yoshizawa, K., Kamada, K., Mizunuma, T., \& Tatenuma, M. (1991). Brewing encyclopedia. Tokyo, Japan: Asakura Publishing Co., Ltd. pp. 406-408.

Obata, A., \& Kuwagaki, D. (2013). Hot seasoning: raw soy sauce. Journal of Cookery Science, 46, 402-404. https://doi.org/10.11402/cookeryscience.46.402

Osuga, A., Iwasaki, Y., Takahashi, T., \& Ogoshi, H. (2013). Effects of the properties of oil and fats on the ease of swallowing mashed potato. Journal of Cookery Science, 46, 15-22. https://doi.org/10.11402/cookeryscience.46.15

Shibata, K., Watanabe, Y., Imamura, M., Obata, A., \& Yasuhara, Y. (2015). Effect of soy sauce seasoning on cooking properties and palatability of flavor-grilled swordfish. Journal of Cookery Science, 48, 301-307. https://doi.org/10.11402/cookeryscience.48.301

Statistical data. (2019). Soy Sauce Information Center. Retrieved from https://www.soysauce.or.jp/statistical-data

Tsuji, R. F., Hamano, M., Koshiyama, D., \& Fukushima, D. (1987). Conditioning of meat with raw soy sauce and its proteinases: their effects on the quality of beef. Journal of Food Science, 52, 1177-1179. https://doi.org/10.1111/j.1365-2621.1987.tb14037.x

Tsuji, S. (1980). Japanese cooking: A simple art. Tokyo, Japan: Kodansha International Ltd. pp. 452-454.

\section{Copyrights}

Copyright for this article is retained by the author(s), with first publication rights granted to the journal.

This is an open-access article distributed under the terms and conditions of the Creative Commons Attribution license (http://creativecommons.org/licenses/by/4.0/). 